\title{
Teratoma during Pregnancy with Positive Estrogen and Progesterone Receptors and Elevated Ca19-9 Antigen Levels
}

\author{
Nilgun Kanlioglu Kuman, ${ }^{1}$ Salih Cokpinar, ${ }^{1}$ Ertan Yaman, ${ }^{1}$ \\ Ibrahim Meteoglu, ${ }^{2}$ and Fisun Karadag ${ }^{3}$ \\ ${ }^{1}$ Department of Thoracic Surgery, Faculty of Medicine, Adnan Menderes University, 09000 Aydin, Turkey \\ ${ }^{2}$ Department of Pathology, Faculty of Medicine, Adnan Menderes University, 09000 Aydin, Turkey \\ ${ }^{3}$ Department of Pulmology, Faculty of Medicine, Adnan Menderes University, 09000 Aydin, Turkey
}

Correspondence should be addressed to Nilgun Kanlioglu Kuman, nilkanlioglu@gmail.com

Received 25 April 2012; Accepted 24 September 2012

Academic Editors: S. Angioni and Y.-B. Tang

Copyright (c) 2012 Nilgun Kanlioglu Kuman et al. This is an open access article distributed under the Creative Commons Attribution License, which permits unrestricted use, distribution, and reproduction in any medium, provided the original work is properly cited.

\begin{abstract}
We present a 27-year-old female patient admitted with an anterior mediastinal mass. She complained of chest discomfort and hemoptysis which began seven months prior. She had given birth five months prior. Thoracic X-ray showed an anterior mediastinal mass. Thorax computed tomography (CT) confirmed a well-defined anterior mediastinal mass with $13 \times 12 \mathrm{~cm}$ diameter, extending to the right hemithorax. It was composed of cystic spaces and discrete areas like soft tissue and fat. Serum Ca 19-9 level was elevated. CT features were consistent with a mature teratoma. During median sternotomy, the tumor revealed adhesions to the right lung and the right subclavian artery. Histologically, the tumor was diagnosed as a mature teratoma. Estrogen and progesterone receptors were detected to be positive in the resected tissue. We conclude that alterations in hormone levels during pregnancy might be the cause of rapid tumor growth which leads to hemoptysis.
\end{abstract}

\section{Introduction}

Early diagnosis of mediastinal tumors is rare since symptoms usually arise after compression of the the adjacent structures [1]. Most of the mediastinal tumors are asymptomatic and $53 \%$ are detected on chest radiography taken for other reasons [2].

\section{Case}

A 27-year-old female patient had been investigated seven months ago because of hemoptysis and chest pain during pregnancy. The chest radiography revealed a $13 \times 12 \mathrm{~cm}$ hypodense lesion with smooth margins at mediastinum. There was no specific feature in her medical history except a full term birth five months ago. Routine laboratory tests and HCG, AFP, CEA, CA 15-3, and prolactin levels were normal whereas CA $19-9$ was elevated $(126 \mathrm{U} / \mathrm{mL})$. CT showed a lobulated lesion filling the anterior mediastinum. Lesion contained a large amount of fat, solid components, and calcifications. The mass extended from right brachiocephalic vein to the right atrioventricular sulcus and was located adjacent to the branches of the aorta and pulmonary veins. There were intraparenchymal small cystic areas and a linear density in the anterior segment of right upper lobe. Thoracic magnetic resonance imaging (MRI) showed a mass with fat, liquid, and solid components, pressing on the heart (Figure 1).

Median sternotomy was performed and a mass lesion with tight adhesions to pericardium, left and right mediastinal pleura, right upper lobe, and brachiocephalic vein was detected in anterior mediastinum. Tumor excision and the right upper lobe wedge resection were performed. During excision, hair, and cartilage tissue protruding from the mass were observed.

Pathological specimen was 443 grams and $13 \times 12 \times 6 \mathrm{~cm}$ in size. Skin, skin appendages, cartilage, bone tissue, bone marrow elements, respiratory, and gastrointestinal epithelium were observed in microscopic examination. Tissue had 

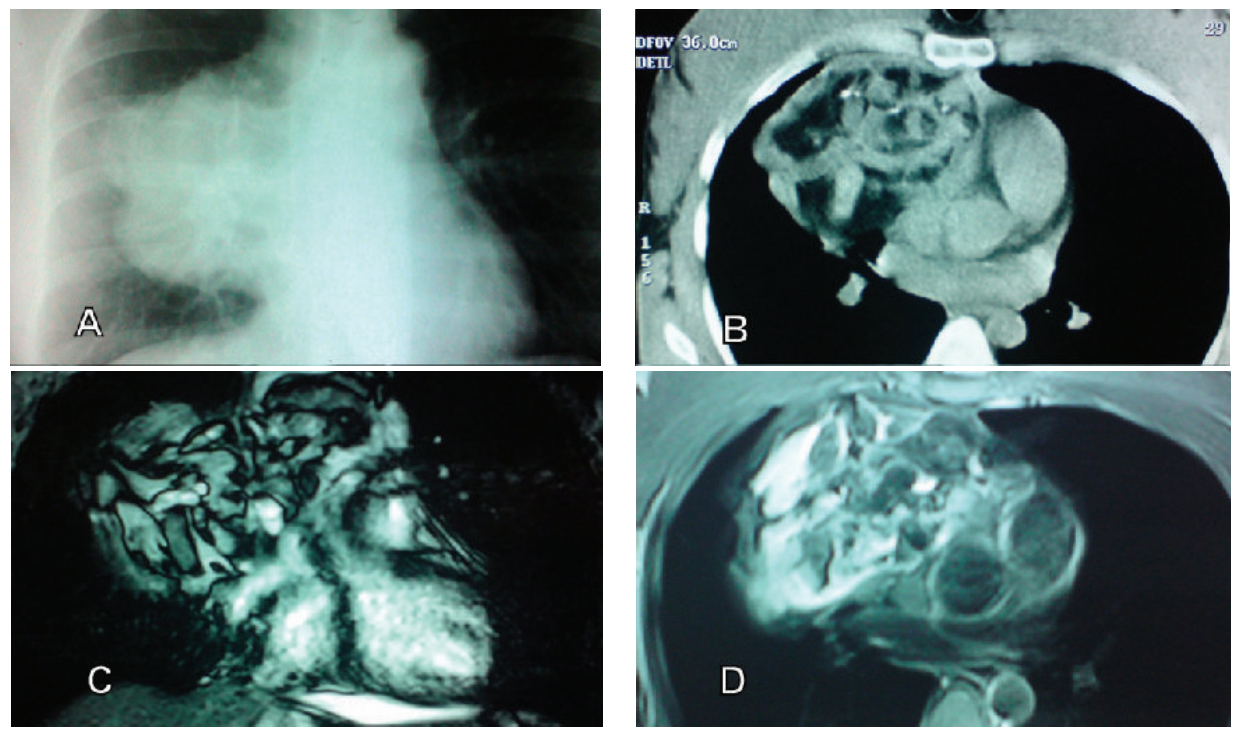

FIgURE 1: (A) Mediastinal lesion on chest radiography. (B) A mass with heterogenous density and calcifications in anterior mediastinum on thorax CT. (C) and (D), heart and main vascular structures are displaced by the mass on thoracic MRI.
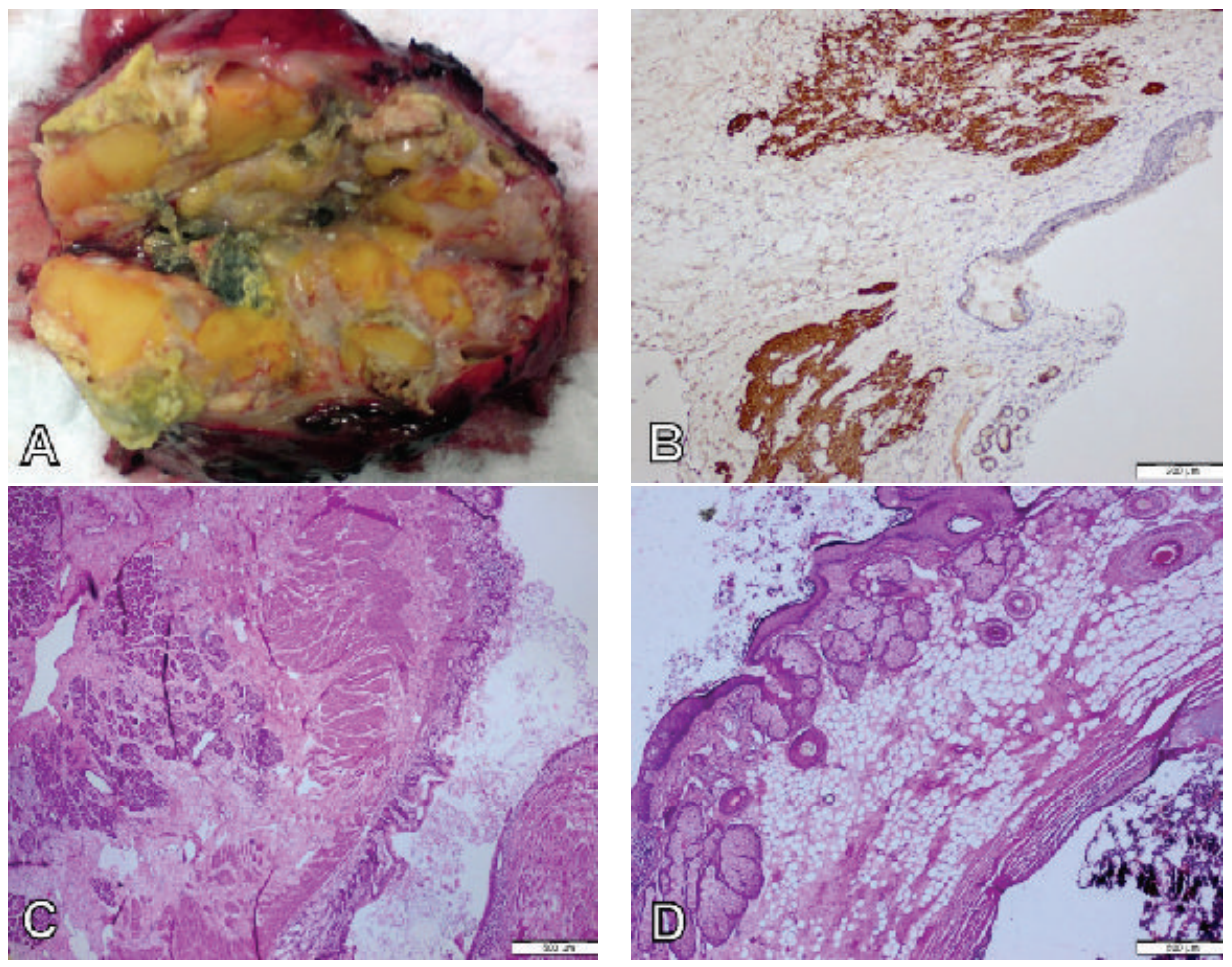

Figure 2: (A) Cystic spaces, fat, cartilage, and hair can be seen in surgical resection material. (B) Glial tissue was stained with GFAP in immunohistochemical study $(\times 40)$. (C) Muscle, connective tissue, columnar epithelium, and pancreatic tissue were seen by microscopy $(\mathrm{HE}, \times 40)$. (D) Epidermis, skin appendages, fat tissue, and bone tissue areas were seen on microscopic study $(\mathrm{HE}, \times 100)$.

mature components, which were stained with glial S100 and GFAP, consistent with teratoma.Emphysematous changes, thickening of alveolar septa, and interstitial mono-nuclear infiltration were detected in the lung specimen, and the surgical margins were tumor free (Figure 2).

Estrogen and progesterone receptors were detected to be positive in the resected tissue (Figure 3 ).
The patient was discharged on postoperative seventh day. There were no complications during 5 months of followup.

\section{Discussion}

Teratomas were described first in 1953 by Willis as a tumor that contained tissues different from the affected organ [3]. 


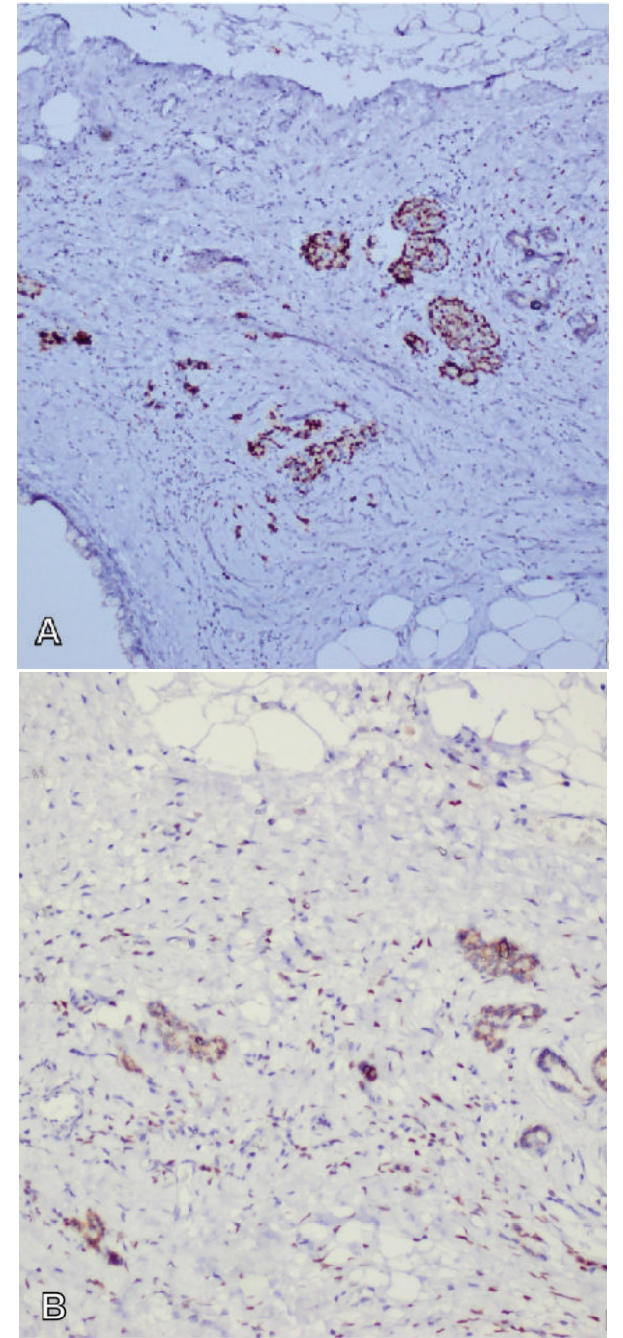

Figure 3: (A) Immunohistochemical PR-positive glandular structures and (B) immunohistochemical ER-positive glandular structures were seen on image $(\times 100)$.

95\% of teratomas are placed in the anterior mediastinum and detected in second frequency after thymomas among the tumors of anterior mediastinum $[1,3]$. Teratomas arise from multipotent primitive embryonic cells and mediastinal teratomas may develop from the surrounding cells of third bronchial cleft [3]. Most teratomas contain mesodermal, endodermal and especially mature ectodermal components [1]. Teratomas can produce hormones because they contain some different cell types [4]. Estrogen receptors and progesterone receptors may be elevated in tissue like our case.

Although most teratomas are asymptomatic, symptoms such as chest pain, dyspnea, and cough may be seen. There is no other case in the literature who became symptomatic during pregnancy and treated after pregnancy like our patient literature. Uyama et al. reported that the alterations in sex hormones in menarche may have played a role in the rapid tumor growth [5]. When teratomas rupture to lung or other adjacent organs, they are always symptomatic. Symptoms have been reported such as hemoptysis, expectoration of hair, and other tissues, pneumonia, acute respiratory distress, pleural empyema. Cardiac tamponade may develop when it ruptured to pericardium [1-3]. The etiology of the perforation is controversial. Ischemia, infection, and inflammation were claimed to be the reasons. In addition, cystic teratomas contain pancreatic or salivary gland tissue. The secreted proteolytic or digestive enzymes may cause adhesion or erosion of the surrounding structures. Rupture or fistula formation may develop as a result of chronic inflammation of the cyst wall [2]. We did not detect complete rupture of the teratoma in our case; a small amount of hemoptysis occurred possibly due to chronic changes as a result of compression of the lungs. However, rupture of teratoma to the lung should be considered in patients presenting with hemoptysis. Although pressure on the heart and great vessels is infrequent, cardiac symptoms such as atrial fibrillation are reported in the literature [6].

Teratomas are seen in anterior mediastinum with about 8-10 cm size, appearing to protrude into a hemithorax on plain chest radiography [3]. CT has diagnostic value for teratomas because of pathognomonic findings of fat and calcific densities [3]. The tumor invasion into surrounding tissues and structural properties can be evaluated with CT [1]. According to CT findings, soft tissue was found in almost all teratomas, while $88 \%$ liquid, $76 \%$ fat, and $53 \%$ calcification were found. MRI is a valuable technique used in determining the relationship with anatomical structures such as mediastinal and hilar vessels and airways [6]. CT and MRI evaluation will be helpful to determine the internal structure of the tumor, the relationship with the surrounding tissue and planning the operation as in our case. Preoperative serum alphafetoprotein (AFP) and B-HCG levels should be evaluated in suspected germ cell tumors [6]. In our case, serum HCG, AFP, CEA, CA 15-3, and prolactin were detected at normal levels but CA 19-9 level was increased. Immunohistochemical studies revealed that epithelial tissues containing squamous epithelium or bronchial components have CA 19-9 [7].

Mature teratomas are treated by surgical resection. Sternotomy is the most preferred method to achieve optimal exposure $[1,6,8]$. Thoracotomy can be the choice when the tumor is localized to one hemithorax [3]. Teratomas have good prognosis and there is no need for adjuvant chemo- or radiation therapy [8]. According to literature, local recurrence rate is low and the total excision is not necessary if dense adhesions were observed to pericardium, lung, or main vascular structures [3]. However, complete resection will enable the correct diagnosis and treatment.

We conclude that alterations in hormone levels during pregnancy such as estrogen and progesterone might have stimulated the sensitive tumor cells leading to tumor growth in our case. Thus, the tumor became symptomatic due to mass effect and increased inflammation led to adhesions to surrounding tissues.

\section{Conflict of Interests}

The authors declare that they have no conflict of interests. 


\section{References}

[1] U. Yetkin, A. Orgencalli, G. Yuncu, and A. Gurbuz, "Large mediastinal teratoma originating from the aortic adventitia," Texas Heart Institute Journal, vol. 31, no. 3, pp. 309-312, 2004.

[2] O. Pikin, K. Kolbanov, V. Kazakevich, and A. Korolev, "Mediastinal mature cystic teratoma perforating into the lung," Interactive Cardiovascular and Thoracic Surgery, vol. 11, no. 6, pp. 827-829, 2010.

[3] O. Soysal, R. Kutlu, K. Saraç, A. Aydin, and S. Ozen, “Teratoma presenting as a cystic lesion on the chest wall," Scandinavian Cardiovascular Journal, vol. 34, no. 5, pp. 543-544, 2000.

[4] T. Tsujimoto, M. Takaichi, H. Endo et al., "A patient with diabetes and breast cancer in whom virilization was caused by a testosterone-producing mature cystic teratoma containing a brenner tumor," American Journal of the Medical Sciences, vol. 341, no. 1, pp. 74-77, 2011.

[5] T. Uyama, Y. Monden, K. Harada et al., "Rapidly growing mature teratoma of the mediastinum: do sex hormones affect growth of the tumor?" Journal of Surgical Oncology, vol. 38, no. 4, pp. 285-289, 1988.

[6] C. Asteriou, N. Barbetakis, A. Kleontas, and D. Konstantinou, "Giant mediastinal teratoma presenting with paroxysmal atrial fibrillation," Interactive Cardiovascular and Thoracic Surgery, vol. 12, no. 2, pp. 308-310, 2011.

[7] M. Kitada, K. Ozawa, K. Sato, Y. Matsuda, S. Hayashi, and T. Sasajima, "Resection of a mediastinal mature teratoma diagnosed owing to sudden chest pain with elevated preoperative serum CA19-9," General Thoracic and Cardiovascular Surgery, vol. 58, no. 6, pp. 298-301, 2010.

[8] D. Karangelis, G. Kalafati, V. Liouras, and N. Tsilimingas, "Germ cell tumors of the mediastinum," Interactive Cardiovascular and Thoracic Surgery, vol. 11, no. 6, p. 829, 2010. 


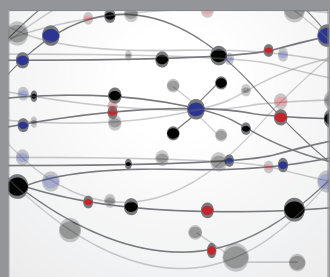

The Scientific World Journal
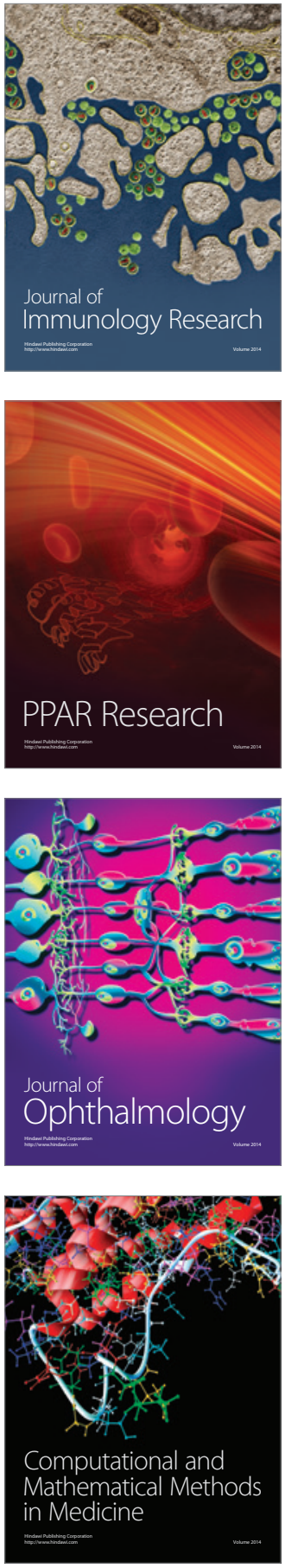

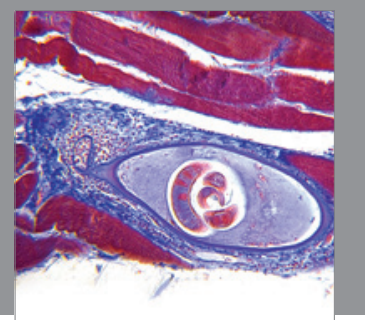

Gastroenterology

Research and Practice
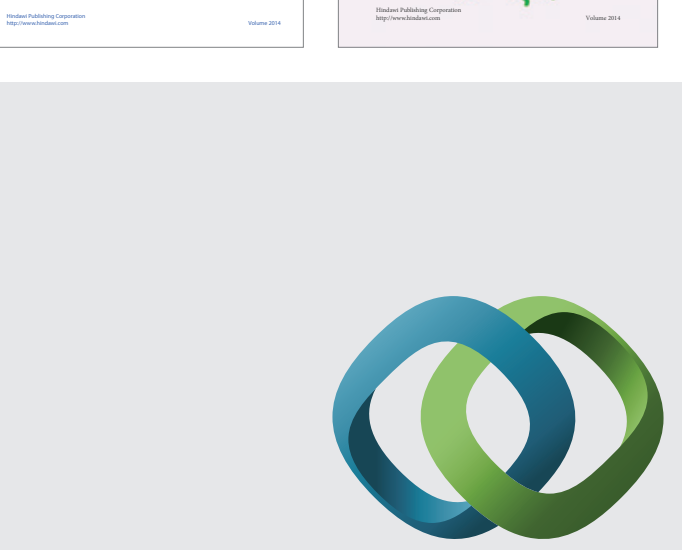

\section{Hindawi}

Submit your manuscripts at

http://www.hindawi.com
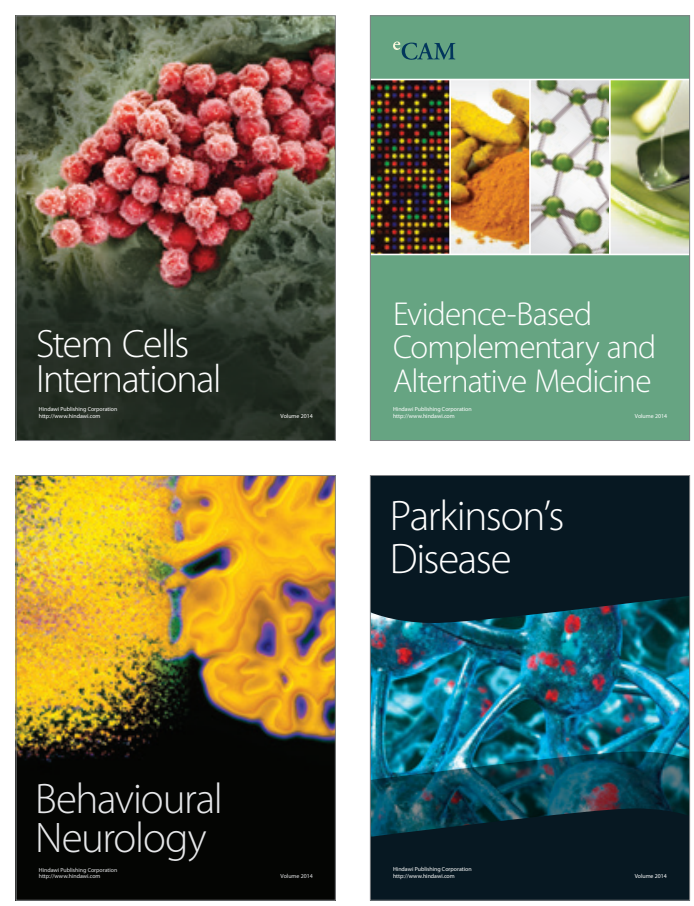

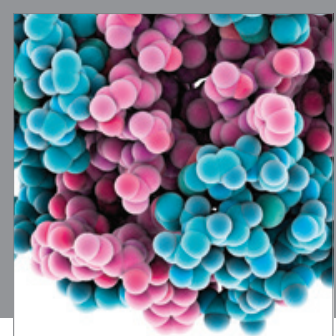

Journal of
Diabetes Research

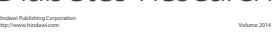

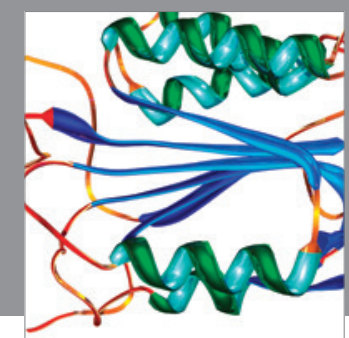

Disease Markers
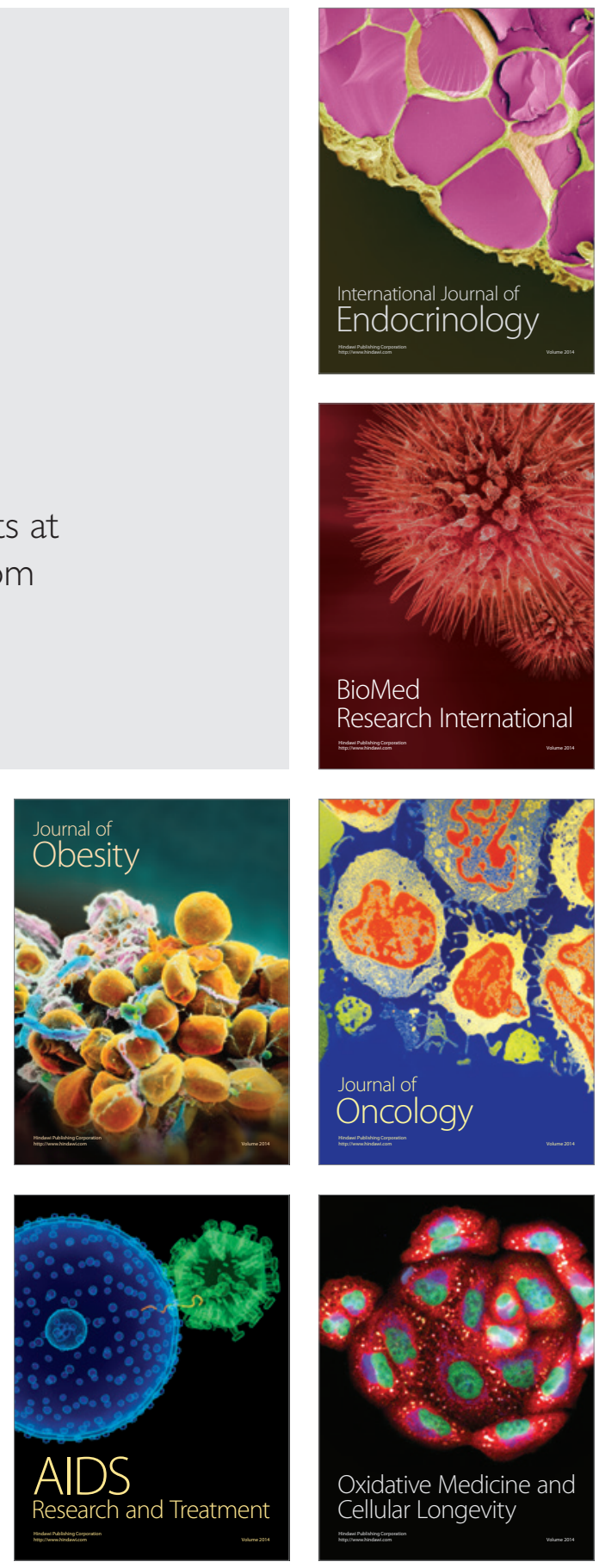